\title{
Adaptive oscillator controller for quadrupled hopping robots
}

\begin{abstract}
As the robots are used in some of the extreme environments such as battle fields, disaster sites or extra planetary exploration, it is necessary to provide robust locomotive strategies that are optimal to each terrain. The hopping gait has been well studied in robotics and proven to be a potential method for locomotion in some of these areas. There have been some difficulties in developing terrain following controllers that maintain robust, steady state hopping gait which is disturbance resistant. This paper discusses controller strategies that have shown the ability to produce the robust hopping gait. An adaptive oscillator controller presented is this paper works remarkably well, both in simulation and experiments. In this paper, novel control methods based on the phase angle are used to control oscillating robotic systems. The phase oscillator uses the sine and cosine of the phase angle to change key properties of a mass-spring-damper system, including amplitude, frequency, and equilibrium. A hopping robot is used to show the applications of this phase oscillator. This paper presents the theoretical framework, simulations and experimental results.
\end{abstract}

Volume 3 Issue 4 - 2019

\author{
Philip New, Andrew Bates, Juan De la Fuente, \\ Thomas Sugar, Sangram Redkar \\ Philip New, Andrew Bates, Juan Da la Fuente, Thomas Sugar, \\ Sangram Redkar
}

Correspondence: Sangram Redkar, The Polytechnic School, Arizona State University, Mesa,Az-86212, USA,

Emailsredkar@asu.edu

Received: June 10,2019 | Published: August 01,2019

Keywords: phase angle, hopping robot, limit cycle, oscillator, controller

\section{Introduction}

An autonomous system is one, which can sense an external event, make some logical choice based on the event, and then respond to the stimulus. In this respect, autonomous robotics has become ubiquitous in modern life. The cost of many consumable products has been greatly reduced through increased production and efficiencies in manufacturing processes, largely due to robotics. Some of the new and most exciting areas where robots are tasked are in Search and Rescue (SAR) scenarios ${ }^{1}$ and military operations to help soldiers with battle field awareness, logistics and physical augmentation..$^{2-14} \mathrm{~A}$ potentially more effective mode of locomotion for rough terrain can be found with legged robotics. Many legged robotic configurations are possible. Control systems are needed to add energy for locomotion in legged robots such as monopedal, bipedal, or quadrupedal robots. Systems can add energy based on negative damping, ${ }_{11}^{11}$ patterns based on phase angles, ${ }^{12,13}$ and impedance control. ${ }^{14}$ In this work, methods have been developed to add energy to assist motion based on adaptive oscillator control. This control method has been shown to work on linear and rotary mechanical systems. This phase-based approach is very powerful because a forcing function can be developed that can act on a hybrid dynamic locomotion system. Even though the dynamics change when the leg is contacting the ground in the stance phase and in the air during the flight phase, the forcing function does not change. It should be noted that in the case of these hopper robots versus some other phase angle controlled systems, the forcing function is not continuously active, rather is activated based on a phase angle and the control objective, i.e. operation in a steady state limit cycle or dampening to zero velocity.

A phase oscillator has been used mainly in wearable robotic systems. A simple application of phase oscillator control is the swingup and balancing of an inverted pendulum. Many approaches have been used to control the system, including energy based control, ${ }^{8-15}$ feedback stabilization, ${ }^{14,15}$ bang-bang ${ }^{2}$ control, ${ }^{16}$ sliding mode control, ${ }^{17-19}$ robust control, ${ }^{20}$ hybrid control, ${ }^{21}$ partial linearization, ${ }^{22,23}$ machine learning, ${ }^{24}$ and simulator-based foresight control. ${ }^{25}$
One of the common control methods is the energy based control or Lyapunov type control method. The energy control method considers the current energy in the system and the total energy needed. When the energy in the system is less than the total energy needed, the control adds energy to the system, usually by providing the full torque in the same direction as the angular velocity. Once the system has enough energy, the control switches to a different method. A problem with energy based control is the quick changes in the forcing function. When the velocity switches from positive to negative, the forcing function switches from full force positive to full force negative. In simulation, quick, large changes are not a problem. But in a real robotic system, quick, large changes can damage the actuator or reduce its life. The phase oscillator can be used for both swing-up and balancing and can create a continuous forcing function that is better for a practical robotic system.

\section{Literature review}

One of the earliest legged walking machines was designed around 1850 by the Russian mathematician Pafnuty Chebyshev. Since then designers, scientists, researchers and inventors have been trying to replicate what nature seems to have done so well. In the last 100 years, a number of systems have been developed into successful machines. ${ }^{17}$ In mid 1980's Marc Raibert showed that hopping is a useful gait for robots with his 3-D prototype and eventually with the "Monopod".? Raibert's designs were mass, spring, and damper systems that used compressed air to act as a spring to produce the effect of both a suspension system and an energy storage device. Zhuang Gao et al., ${ }^{19}$ also made the argument that legged robots can navigate terrain that wheeled or tracked vehicles have trouble with. They surveyed many commercially available heavy duty legged robots to assess the state of performance in these types of robots. The mission of the robots, they discussed, ranged from forestry to military to space exploration; these included the Plustech Walking Machine ${ }^{20}$, Boston Dynamics Big $\operatorname{Dog}^{21}$ and LS3,22 and NASA's ATHELETE. ${ }^{23}$

They concluded that these robots could be well suited for outdoor exploration and disaster relief but many problems still exist. There are 
adverse efficiencies typically from poor leg design. There is a strong need for compliance and in most cases it is produced virtually instead of direct compliance. Virtual compliance is the use of force sensors in an end effector to allow a robot to move in the direction of an externally applied force, ${ }^{24,25}$ This gives the appearance of compliance or a soft touch. There is also a need to reduce computational complexity in the control algorithm. This problem appears to be common between both heavy-duty legged robots and their smaller counterparts. ${ }^{26-28}$ Zhuang specifically suggested that further research should aim to develop a simpler intelligent control algorithm that meets compliance requirements. In a study published in 2010, hopping in humans and robots were studied. ${ }^{29}$ The authors suggested that current research has used apex-preserving hopping as the model for human and robotic hopping. In their opinion this model should be replaced with a spring, mass, damper system that utilized a constant energy supply. A constant energy supply means that every cycle of the gait receives the same energetic input no matter what are the states of the hopper. As system experiences dissipative losses of energy from damping and friction, the energy injection, in theory, will compensate for the losses.

The paradigm of the hopping model as apex-preserving to one of terrain following will result in a more robust, disturbance resistant hopper and one which is less computationally demanding. They suggest that the energy dissipation itself is an essential component to a stable, steady state hopping instead of being an intrinsic perturbation to the system. A question that comes out of the paper is "does there exist a solution that guarantees stable hopping to produce robust and stable terrain following locomotion?" We propose that the Hill-type muscle model may produce the desired result. This model utilizes a force-length and force-velocity relationship to achieve stability and robustness from perturbations. It also uses a contractive element along with both a series and parallel elastic element. ${ }^{30,31} \mathrm{~A}$ mechanism, if it existed, also could use these relationships and elements to produce a system with the same stability as the model. Many similarities exist between the Hill-type muscle model and the phase oscillator control system discussed in this paper. The most important similarity is the result of a stable controller that is resistant to disturbances and converges to a steady state limit cycle independent of initial conditions.

\section{Methodology}

Phase plane analysis can be studied to determine if the system shows limit cycle. ${ }^{32}$ In this work, a non-linear forcing function based on the sine of the phase angle is used to drive system to limit cycle. Because the sine function can never be greater or smaller than one or negative one, the function is bounded.

A standard, second order, mechanical system equation is given by equation (1) where $m$ represents the mass, $b$ represents the damping, $k$ represents the stiffness.

$$
m \ddot{x}+b \dot{x}+k x=0
$$

The natural frequency of oscillations of the system modeled by Equation (1) is dependent on the mass, $\mathrm{m}$, and spring constant, $k$. The behavior of the amplitude of the oscillations is dependent on the damping coefficient, $\mathrm{b}$. If $b>0$, the system has positive damping, the oscillations will shrink and disappear over time. If $b>0$, the system has negative damping, and the oscillations will continue to grow. If $b=0$, the system has no damping, and the oscillations will remain at a constant amplitude.
Researchers have added negative damping to systems to force a system to move, but the negative damping can become unstable because $c \dot{x}$ grows as the velocity gets larger.

$$
m \ddot{x}+b \dot{x}+k x=c \dot{x}
$$

A simpler method of control is a phase oscillator. A phase oscillator adds energy to the system based on the phase angle of the system. Figures $1 \& 2$ shows the phase angle, $\phi$, on a phase plot. $\phi$ is defined by

$$
\phi=\operatorname{atan} 2(\dot{x}, x)
$$

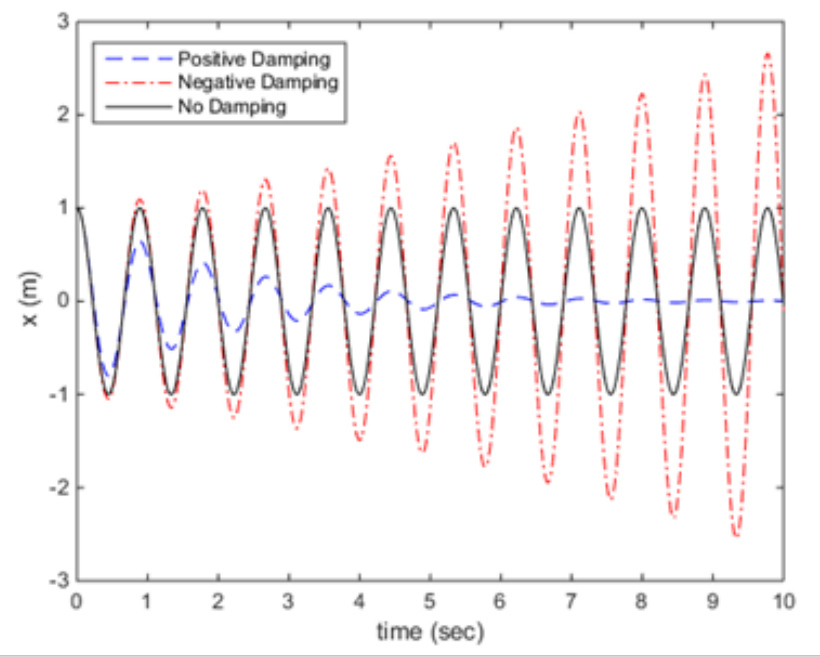

Figure I Spring response due to positive, negative, and no damping. $m=1$ $\mathrm{kg}, k=50 \mathrm{~N} / \mathrm{m}, b$ is in $\mathrm{Ns} / \mathrm{m}$, initial position $=1 \mathrm{~m}$, initial velocity $=0 \mathrm{~m} / \mathrm{s}$.

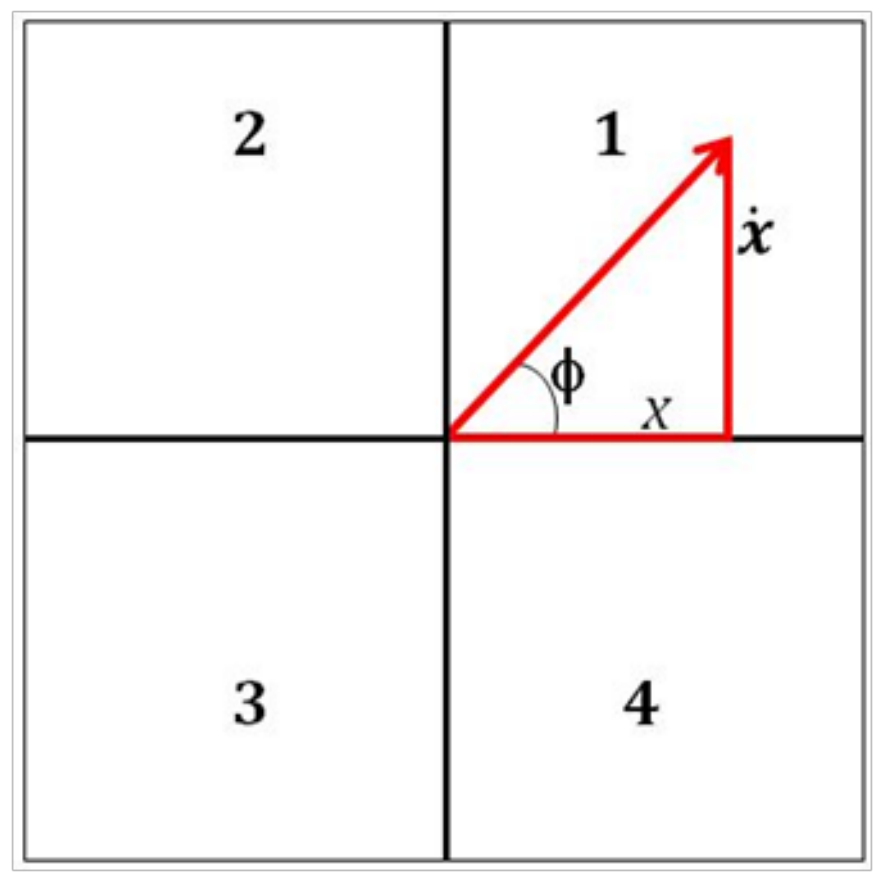

Figure 2 Phase plot definition, horizontal axis is the position, $x$, and the vertical axis is the velocity, $\dot{x}$. The sine of the phase angle phi is given by the opposite over the hypotenuse. The hypotenuse is given by $\sqrt{\dot{x}^{2}+x^{2}}$. 
The phase angle can be used to determine when to add energy to the system and how much energy to add. The phase angle remains close to $\pm \frac{\pi}{2}$ for the majority of each oscillation. When the velocity is divided by the natural frequency (defined in Equation 4), the phase angle (redefined in Equation 5), allows for more precise phase oscillator controls (Figures 3-6).

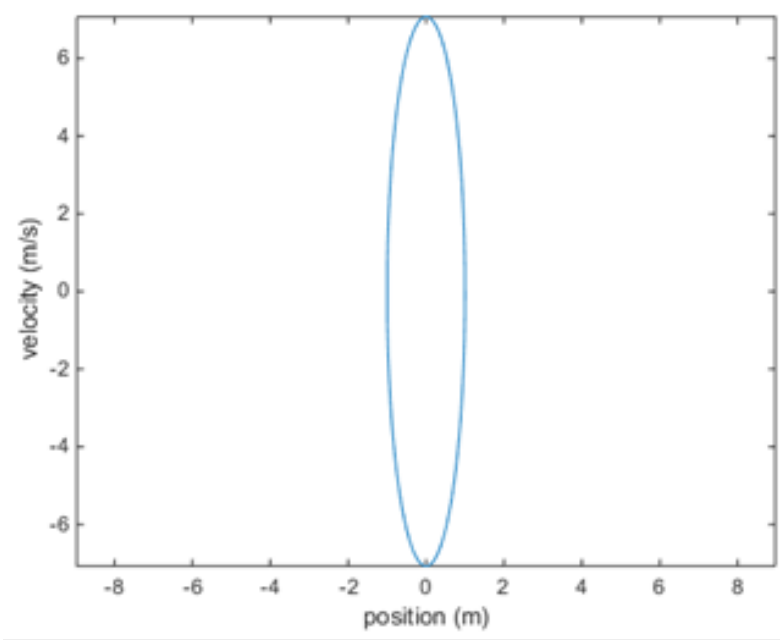

Figure 3 Phase plot of an oscillating system with no damping using Equation 3 for $\phi$.

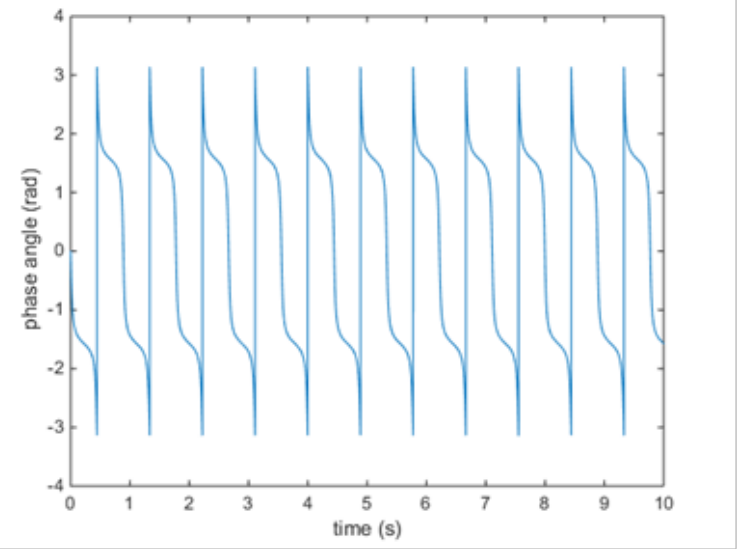

Figure 4 Phase angle of an oscillating system with no damping using Equation 3 for $\phi$.

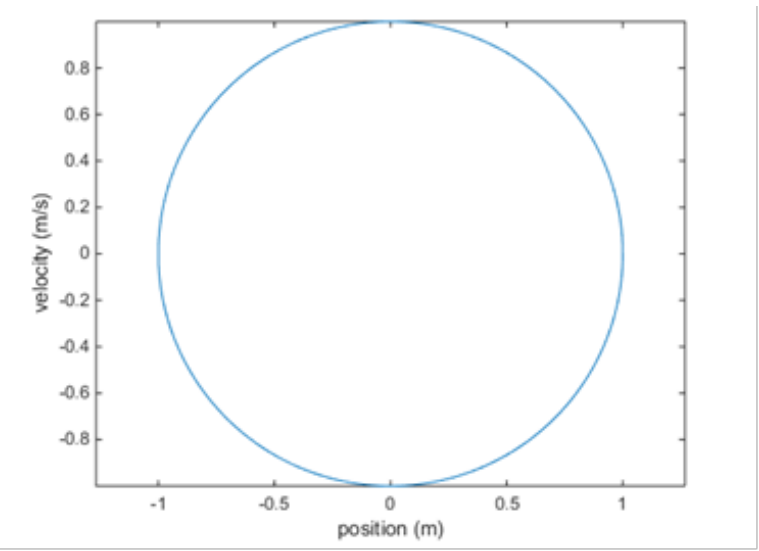

Figure 5 Phase plot of an oscillating system with no damping Equation 5 for $\phi$

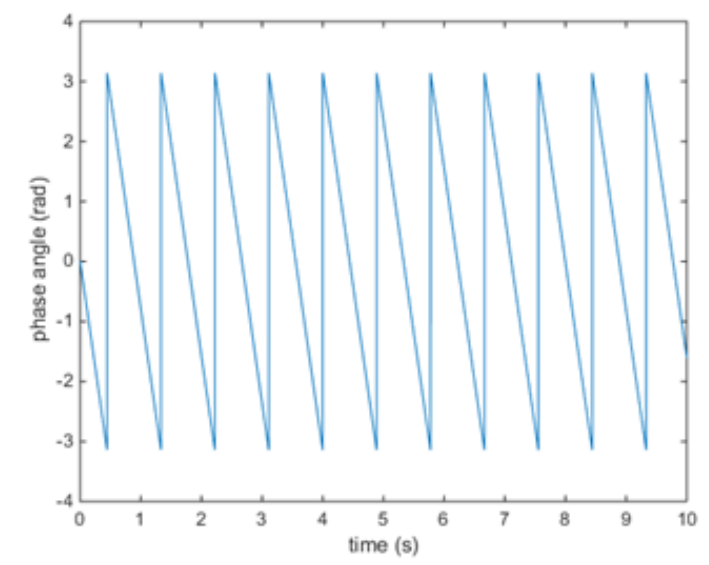

Figure 6 Phase angle of an oscillating system with no damping using Equation 5 for $\phi$.

$$
\begin{aligned}
\omega & =\sqrt{\frac{k}{m}} \\
\phi & =\operatorname{atan} 2\left(\frac{\dot{x}}{\omega}, x\right)
\end{aligned}
$$

The control system described here adds energy to the mechanical system by using the phase oscillator and the equation is given by

$$
\ddot{m} x+b \dot{x}+k x=c \sin (\phi)
$$

Figure 7 shows the system response with damping and phase oscillator. In this case, the oscillations converge to a limit cycle. This limit cycle can be found by solving Equation 6 analytically. The sine of the phase angle is defined by Equation 7 . The steady-state solution of Equation 6 is given by Equations 8, 9, and 10 as shown below.

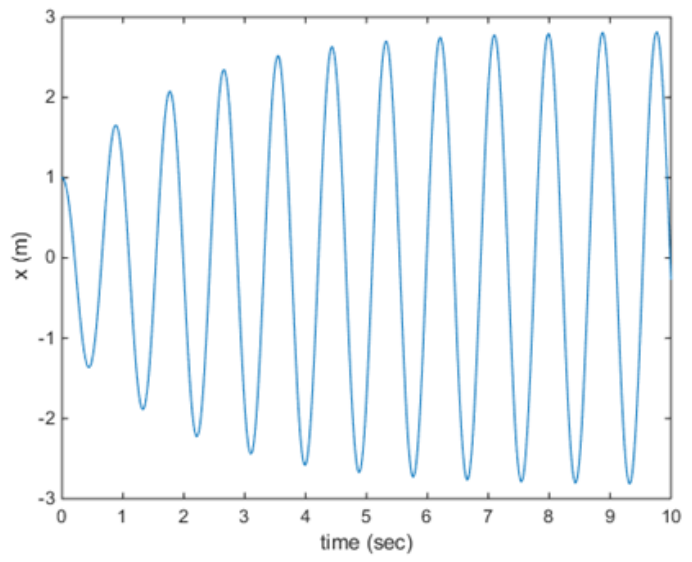

Figure 7 Spring response with phase oscillator. $m=1 \mathrm{~kg}, b=1 \mathrm{Ns} / \mathrm{m}, k=$ $50 \mathrm{~N} / \mathrm{m}, c=20 \mathrm{~N}$, initial position $=1 \mathrm{~m}$, initial velocity $=0 \mathrm{~m} / \mathrm{s}$.

$$
\begin{aligned}
& \sin \phi=\frac{\left(\frac{\dot{x}}{\omega}\right)}{\sqrt{\left(\frac{\dot{x}}{\omega}\right)^{2}+x^{2}}} \\
& x(t)=A \sin (\omega t) \\
& \dot{x}(t)=A \omega \cos (\omega t) \\
& \ddot{x}(t)=-A \omega^{2} \sin (\omega t)
\end{aligned}
$$


Substituting equations 8 and 9 into equation 10 yields equation 11 .

$\sin \phi=\frac{\left(\frac{A \omega \cos (\omega t)}{\omega}\right)}{\sqrt{\left(\frac{A \omega \cos (\omega t)}{\omega}\right)^{2}+A \sin (\omega t)^{2}}}=\frac{A \cos (\omega t)}{A \sqrt{(\cos (\omega t))^{2}+(\sin (\omega t))^{2}}}=\cos (\omega t)$

Equations 8, 9, 10, and 11 can be substituted into Equation 6, as shown in Equation 12, to show the dependence of the amplitude of the system, $A$, on the coefficient $C$.

$$
\begin{aligned}
& -m A \omega^{2} \sin (\omega t)+b A \omega \cos (\omega t)+k A \sin (\omega t)=c \cos (\omega t) \\
& k-m \omega^{2}=0 \\
& c=b A \omega \\
& A=\frac{c}{b \omega}
\end{aligned}
$$

Equations 14 and 15 show the relationship between C and A. Using the sine of the phase angle as a forcing function can be thought of as adding another damping term to the system. A positive coefficient in Equation 6 gives negative damping, as the forcing function is on the right-hand side of the equation, adding energy to the system. Unlike the damping provided by the term $b \dot{x}$, the damping provided by $c \sin \phi$ is bounded in the range of $[-c, c]$. Also, the damping of the phase oscillator varies from $-c$ to $C$ even when $\dot{x}$ is very small. The behavior of the bounded damping can explain intuitively the steadystate solution given by Equation 8 . When $\dot{x}$ is large, the positive damping from $b \dot{x}$ removes more energy from the system than the negative damping from $c \sin \phi$ adds to the system. When $\dot{x}$ is small, the positive damping from $b \dot{x}$ removes less energy from the system than the negative damping from $c \sin \phi$ adds to the system. In either case, the system is pushed toward the same limit cycle, as seen in Figure 7. While giving the sine term with a positive coefficient can be thought of as negative damping, a negative coefficient can be thought of as positive damping, removing energy from the system. Figure 8 shows energy being removed from a system by a phase oscillator.

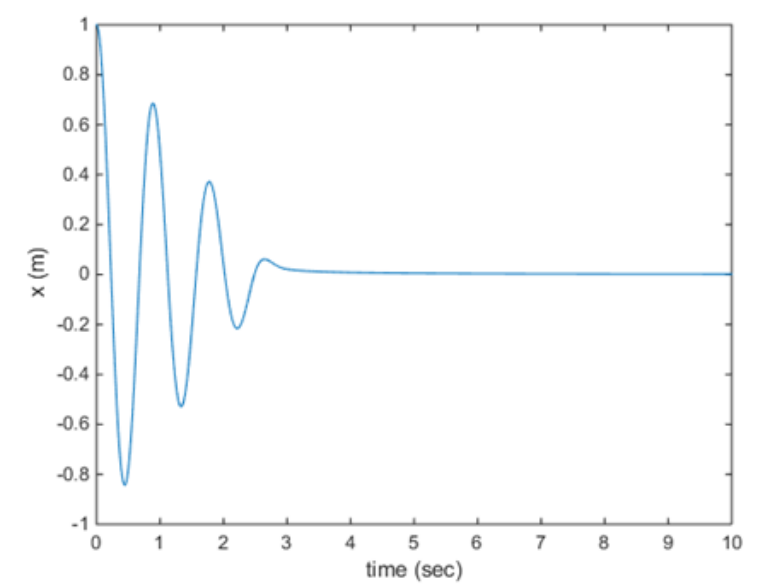

Figure 8 Spring response with phase oscillator. $m=\mathrm{l} \mathrm{kg}, b=0 \mathrm{Ns} / \mathrm{m}, k=$ $50 \mathrm{~N} / \mathrm{m}, c=-5 \mathrm{~N}$, initial position $=1 \mathrm{~m}$, initial velocity $=0 \mathrm{~m} / \mathrm{s}$.

Equation 16 gives the definition of the cosine of the phase angle. The cosine of the phase angle has $x$ in the numerator instead of $\frac{\dot{x}}{\omega}$ , meaning using cosine instead of sine as a forcing function will act as a spring instead of damping. Equations 17 to 20 show the steady state solution from Equation 8 which is still valid for a system with no damping.

$\cos \phi=\frac{x}{\sqrt{\left(\frac{\dot{x}}{\omega}\right)^{2}+x^{2}}}=\frac{A \sin (\omega t)}{\sqrt{\left(\frac{A \omega \cos (\omega t)}{\omega}\right)^{2}+A \sin (\omega t)^{2}}}=\frac{A \sin (\omega t)}{A \sqrt{(\cos (\omega t))^{2}+(\sin (\omega t))^{2}}}=\sin (\omega t)$

$$
\begin{aligned}
& m \ddot{x}+k x=\cos \phi \\
& -m A \omega^{2} \sin (\omega t)+k A \sin (\omega t)=c \sin (\omega t) \\
& \left(k-m \omega^{2}\right) A=c \\
& \omega^{2}=\frac{k-\frac{c}{A}}{m}
\end{aligned}
$$

A negative coefficient of the cosine of the phase angle results in a normal spring, which "pulls" toward $x=0$. Similar to the sine term, the force is bounded by and varies betwee $[-c, c]$. A positive coefficient results in a spring which "pushes" away from $x=0$, but pushes with more force as the displacement increases. Figure 9 shows how a system (without damping) changes with the addition of a phase oscillator using the cosine of the phase angle. As $C$ is negative, the phase oscillator acts as a spring, increasing the frequency. The increased frequency can be explained mathematically by Equation 20 .

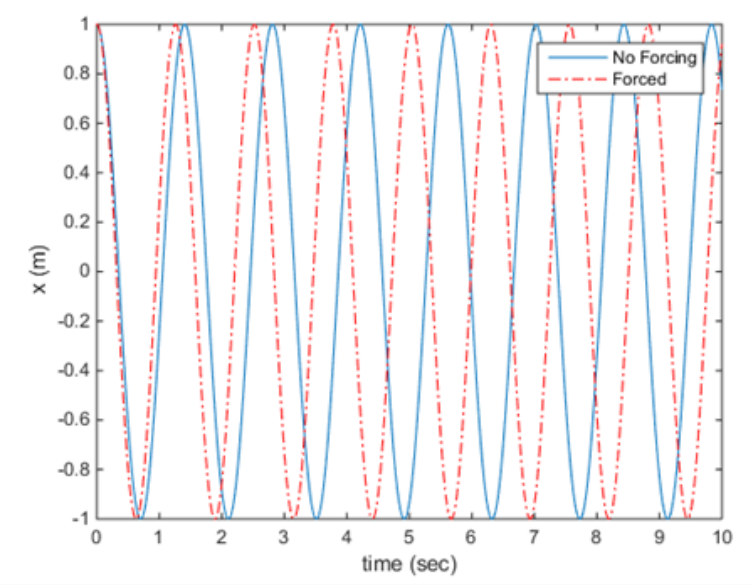

Figure 9 Spring response without and with phase oscillator (cosine). $m=$ I $\mathrm{kg}, b=0 \mathrm{Ns} / \mathrm{m}, k=20 \mathrm{~N} / \mathrm{m}, c==-5 \mathrm{~N}$, initial position = $1 \mathrm{~m}$, initial velocity $=0 \mathrm{~m} / \mathrm{s}$.

The phase oscillator can use both the sine of the phase angle and the cosine of the phase angle simultaneously to control both the amplitude and the frequency of an oscillating system. Equation 21 shows a system forced by a phase oscillator using both the sine and cosine of the phase angle. The system can still be solved with Equation 8, as shown by Equations 22 through 24.

$$
\begin{gathered}
m \ddot{x}+b \dot{x}+k x=c_{1} \sin \phi+c_{2} \cos \phi \\
-m A \omega^{2} \sin (\omega t)+b A \omega \cos (\omega t)+k A \sin (\omega t)=c_{1} \cos (\omega t)+c_{2} \sin (\omega t)
\end{gathered}
$$

$$
\begin{aligned}
& b A \omega=c_{1} \\
& \left(k-m \omega^{2}\right) A=c_{2}
\end{aligned}
$$


Equations 23 and 24 allow the designer to choose any amplitude and frequency. The $\omega$ shown is the frequency of the solution, not necessarily the natural frequency of the system (Figure 10). Using both sine and cosine in the phase oscillator is useful for visualizing the damping and spring components, separately. However, the two terms can be combined into a single term for sim ${ }^{-1}$ icity. Figure 11 and Equations 25 through 27 define the phase shift ${ }^{\alpha}$ and the amplitude $U$. Equations 28 through 30 show the simplification of the phase oscillator.

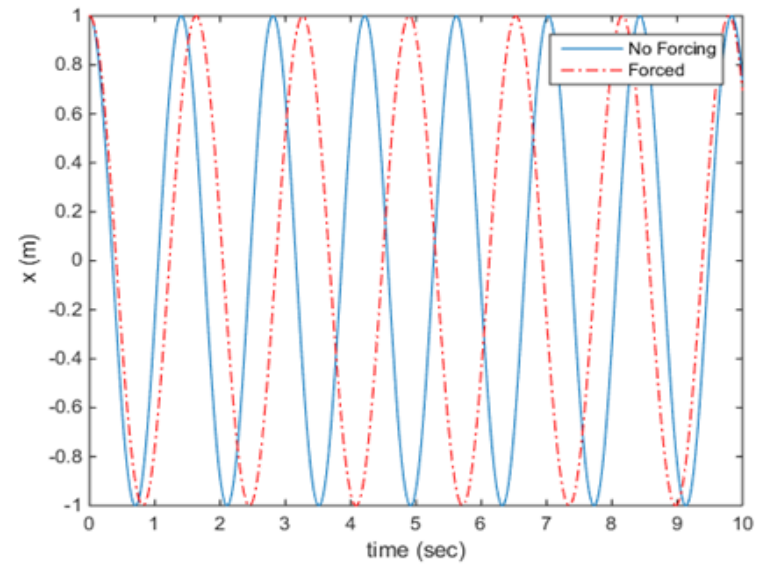

Figure 10 Spring response without and with phase oscillator (cosine). $m=$ I $\mathrm{kg}, b=0 \mathrm{Ns} / \mathrm{m}, k=20 \mathrm{~N} / \mathrm{m}, c==5 \mathrm{~N}$, initial position = I m, initia velocity $=0 \mathrm{~m} / \mathrm{s}$.

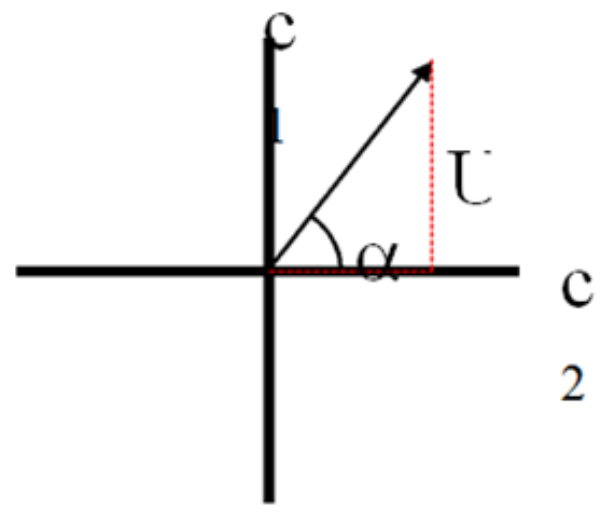

Figure II $\alpha$, in relation to $c_{1}$ and $c_{2}$

$$
\begin{aligned}
& \sin \alpha=\frac{c_{1}}{\sqrt{c_{1}^{2}+c_{2}^{2}}} \\
& \cos \alpha=\frac{c_{2}}{\sqrt{c_{1}^{2}+c_{2}^{2}}} \\
& U=\sqrt{c_{1}^{2}+c_{2}^{2}}
\end{aligned}
$$$$
c_{1} \sin \phi+c_{2} \cos \phi=\sqrt{c_{1}^{2}+c_{2}^{2}}\left(\frac{c_{1}}{\sqrt{c_{1}^{2}+c_{2}^{2}}} \sin \phi+\frac{c_{2}}{\sqrt{c_{1}^{2}+c_{2}^{2}}} \cos \phi\right)
$$

$c_{1} \sin \phi+c_{2} \cos \phi=U(\sin \alpha \sin \phi+\cos \alpha \cos \phi)$

$c_{1} \sin \phi+c_{2} \cos \phi=U \cos (\phi-\alpha)$
The balance between damping and spring provided by the phase oscillator depends on the phase shift of the forcing function, $\alpha$. The maximum value reached by the forcing function is given by the amplitude, $U$. Figure 12 shows the spiral staircase behavior of the phase shift $\alpha$, bounded by the interval $[-\pi, \pi]$. Figure 13 shows the conical behavior of the amplitude $U$ which is bounded by the interval $[0, \infty)$. Using sine of phase angle as the forcing function, can change the amplitude of the oscillations directly based on the $c$ value. But, it is not possible to control the frequency of the oscillations as it depends only on system's natural frequency. On the other hand, using cosine of the phase angle as a forcing function can only affect the frequency of the system during transitions but cannot generate a limit cycle. Therefore, using both sine and cosine of the phase angle can control both the amplitude and frequency of the oscillations based on the values $c_{1}$ and $c_{2}$.

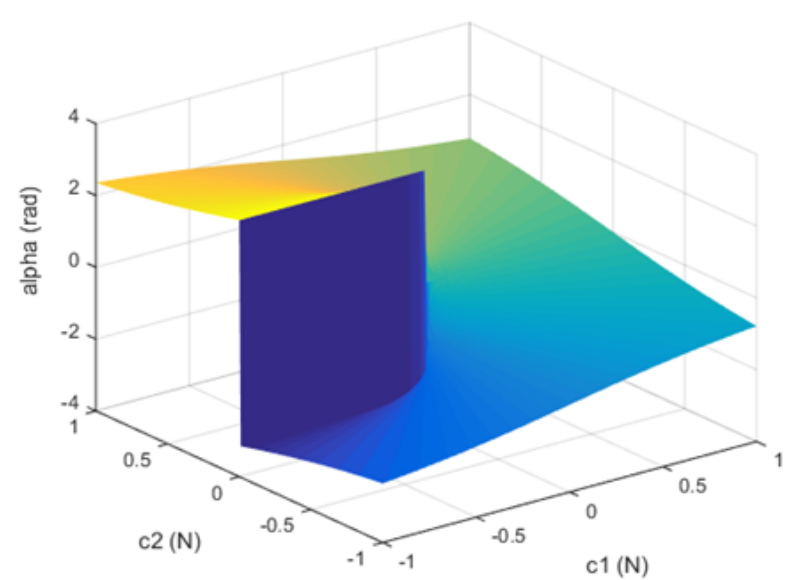

Figure 12 Phase shift $\alpha$, as $c_{1}$ and $c_{2}$ vary between $-\mathrm{I}$ and I.

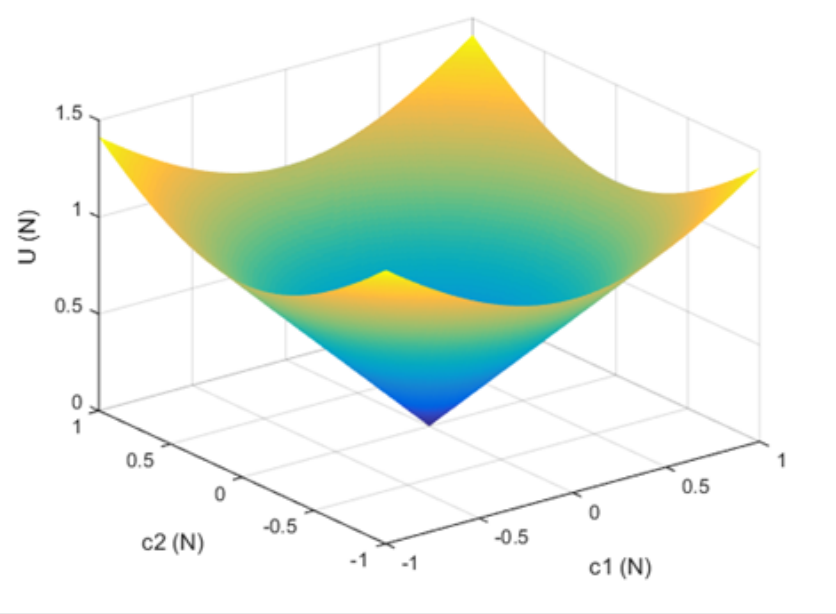

Figure 13 Amplitude $U$, as $c_{1}$ and $c_{2}$ vary between $-\mathrm{I}$ and $\mathrm{I}$.

The mechanical system put represented as a general second order system is :

$f(x) \equiv \ddot{x}=-\frac{b}{m} \dot{x}-\frac{k}{m} x+\frac{c_{1} \dot{x}+c_{2} \omega x}{\sqrt{\omega^{2} x^{2}+\dot{x}^{2}}}=-2 \zeta \omega_{n} \dot{x}-\omega_{n}^{2} x+\frac{c_{1} \dot{x}+c_{2} \omega x}{\sqrt{\omega^{2} x^{2}+\dot{x}^{2}}}$

The existence of the limit cycle can be evaluated with the PoincarèBendixson criterion. Using the continuously differentiable function of Equation 32, its gradient is shown in Equation 33. The product of the 
system in Equation 31 and the gradient of of Equation 33 is presented in Equation 34.

$$
\begin{aligned}
& V(x)=\frac{\omega^{2} x^{2}}{2}+\frac{\dot{x}^{2}}{2} \\
& \nabla V(x)=\omega^{2} x \dot{x}+\ddot{x} \ddot{x} \\
& f(x) \cdot \nabla V(x)=\left(\omega^{2}-\omega_{n}^{2}\right) x \dot{x}-2 \zeta \omega_{n} \dot{x}^{2}+\frac{c_{1} \dot{x}^{2}+c_{2} \omega x \dot{x}}{\sqrt{\omega^{2} x^{2}+\dot{x}^{2}}}
\end{aligned}
$$

According to the Poincarè-Bendixson criterion it is possible to determine a region $M=\left\{K_{1} \leq V(x) \leq K_{2}\right\}$ where $0 \leq K_{1} \leq K_{2}, M$ is closed and bounded, in which it is guaranteed the existence of a closed orbit. The vector field points inwards for $F(x) \cdot \nabla V(x)<0$ , outwards for $F(x) \cdot \nabla V(x)>0$, and tangent for $F(x) \cdot \nabla V(x)=0$ Consequently for this system the vector field points inwards in Equation 35, outwards in Equation 36, and is tangent in Equation 38.

$$
\begin{aligned}
& \frac{c_{1} \dot{x}+c_{2} \omega x}{\sqrt{\omega^{2} x^{2}+\dot{x}^{2}}}<2 \zeta \omega_{n} \dot{x}+\left(\omega^{2}-\omega_{n}^{2}\right) x \\
& \frac{c_{1} \dot{x}+c_{2} \omega x}{\sqrt{\omega^{2} x^{2}+\dot{x}^{2}}}>2 \zeta \omega_{n} \dot{x}+\left(\omega^{2}-\omega_{n}^{2}\right) x \\
& \frac{c_{1} \dot{x}+c_{2} \omega x}{\sqrt{\omega^{2} x^{2}+\dot{x}^{2}}}=2 \zeta \omega_{n} \dot{x}+\left(\omega^{2}-\omega_{n}^{2}\right) x
\end{aligned}
$$

Therefore we get the closed orbit

$$
\omega^{2} x^{2}+\dot{x}^{2}=\left(\frac{c_{1} \dot{x}+c_{2} \omega x}{2 \zeta \omega_{n} \dot{x}+\left(\omega^{2}-\omega_{n}^{2}\right) x}\right)^{2}
$$

$$
\text { Consequently }\left\{K_{1}=V(x)=K_{2}\right\} \text {, }
$$

$V(x)=\frac{1}{2}\left(\frac{c_{1} \dot{x}+c_{2} \omega x}{2 \zeta \omega_{n} \dot{x}+\left(\omega^{2}-\omega_{n}^{2}\right) x}\right)$. The limit cycle exist for $\frac{c_{1} \dot{x}+c_{2} \omega x}{2 \zeta \omega_{n} \dot{x}+\left(\omega^{2}-\omega_{n}^{2}\right) x}>0$. The orbit is well defined for $c_{1}=\rho 2 \zeta \omega_{2}$, $c_{1}=\rho 2 \zeta \omega_{2}, c_{2}=\frac{\rho}{\omega}\left(\omega^{2}-\omega_{n}^{2}\right)$, where $\rho$ is a proportionality variable that defines the radius of the orbit. This proportionality variable depends on the design parameters for frequency and amplitude of the oscillations as seen in Equation 23 and Equation 24. Solving these equations for the general second order dynamic system, the value of amplitude is defined in Equation 39 and the frequency of the oscillations in Equation 40.

$$
\begin{aligned}
& A=\frac{c_{2} \zeta+\sqrt{c_{1}^{2}+\zeta^{2} c_{2}^{2}}}{2 \omega_{n}^{2} \zeta} \\
& \omega=\frac{-c_{2} \zeta \omega_{n}+\omega_{n} \sqrt{c_{1}^{2}+\zeta^{2} c_{2}^{2}}}{c_{1}}
\end{aligned}
$$

The phase based oscillator controller can operate as non-linear controller using error $\theta$ as the variable of interest, which is equal to the difference $e=x_{d}-x$, where $x_{d}$ is the desired value of $\mathrm{x}$. The forcing function for the controller is defined as:

$$
F=\frac{c_{1} \dot{e}+c_{2} \omega e}{\sqrt{e^{2}+\omega^{2} e^{2}+n}}
$$

Where $0<\mathrm{n}<<1$. The use of $\mathrm{n}$ will avoid indefinite values for controller. The forcing function will drive the error to zero for values of $c_{1}<0$. Using $c_{2}=0$ will oscillate the error to zero at the natural frequency of the system, using $c_{2}<0$ reduces the response time and the stabilization time. In order to compare the performance of phase oscillator controller with linear PID controller, simulations were performed on the example system to follow the step reference at 2 seconds, and 3 perturbations were introduced. The PID controller has the structure given by Equation 42

$$
F=K_{p} e+K_{d} e+K_{i} e
$$

The PID controller is tuned with MATLAB tuning toolbox, the gains $K_{p}=317, K_{i}=505$ and $K_{d}=34.34$ are used. For phase controller, the values for $c_{1}=-1000$ and $c_{2}=-8000$ are used. The results of the simulation are shown in Figure 13. Although both the controller showed similar performance, the phase oscillator controller exhibited slightly faster response than PID.

\section{Hopping robot}

The hopping robot is shown in Figure 14. A pneumatic cylinder is used as the actuator with spring ( 7 pounds/inch or $1.25 \mathrm{k}$ newton/ meter) that was used for direct compliance and to return energy to the system as it hopped. The leg is attached to the body through a rotary joint and a four bar linkage system with the motors. The four bar linkage system can be seen in Figure 15. The linear strip (Figure 16) was secured between two plates. It was decided that the one end of the plates could be attached to the spring retainer. The spring retainer was secured in place at the end of the cylinder. A notch was added to the hip and a grove was cut into the inner linear strip plate to ensure that the linear strip was always in the proper place to be read by the encoder. This feature is not shown in Figure 16. A bronze sleeve bearing was installed inside the hip. This allowed the cylinder and the hip to slide freely past one another. The spring is also pressed into the hip. The other end of the spring was pressed against the spring retainer which is fixed to the bottom of the cylinder. All parts were made with 6061 aluminums except for the acrylic inner linear strip plate and the polymer linear strip bracket. The actuators were Numatics $M$ Series 1-1/16" spring return and were deployed using Numatics LO1 Series valves. The valves were mounted to the side walls of the body, positioned in between the motors. The original design for control of the robot was with the Cerebot MC-7 board which uses a Microchip Pic-33 processor. This was eventually changed to an Advantech PC104. The digital input and output used a Sensoray 526 board. The PC-104 was eventually chosen because of its ability to read in four encoders simultaneously. Figure 17 shows the component block diagram for easy visualization of the quadruped robot system.

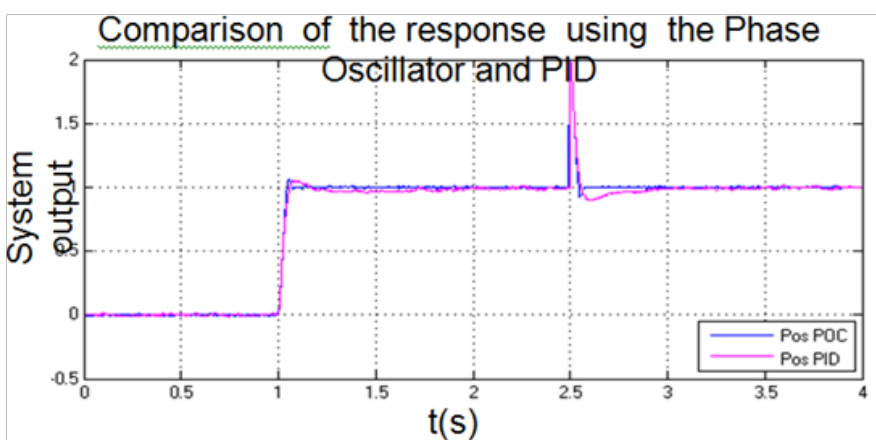

Figure I4 System response with the two controllers. In blue, the response using the phase based oscillator controller. In pink, the response using the PID controller. 


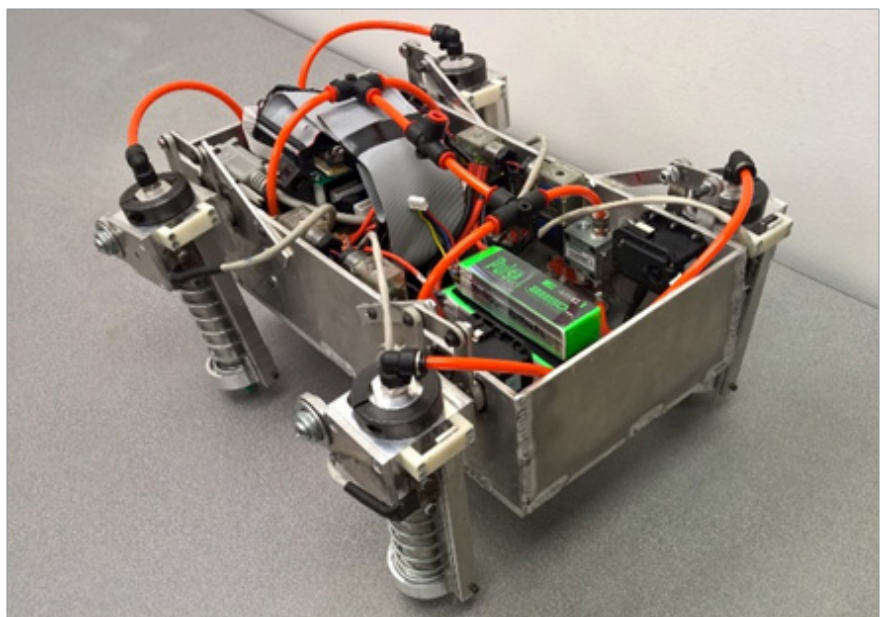

Figure 15 Experimental 4DOF hopping robot.

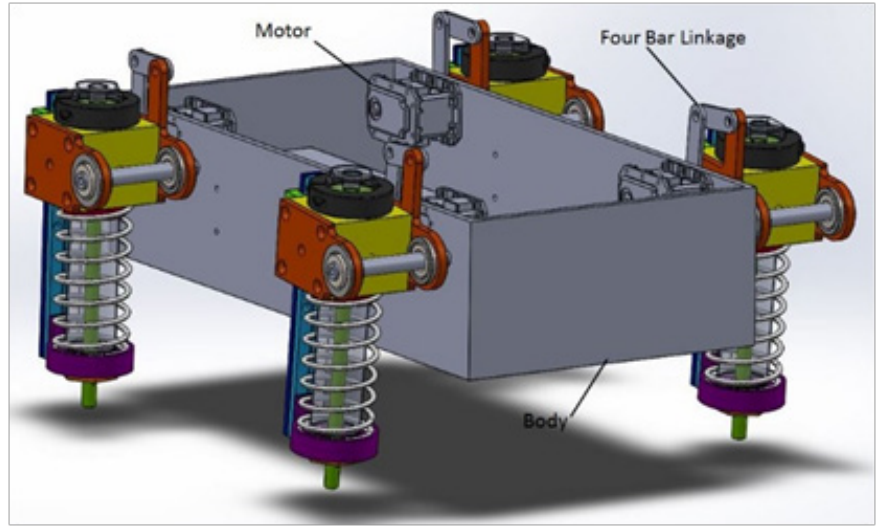

Figure 16 Leg design of the 4 DOF hopping robot.

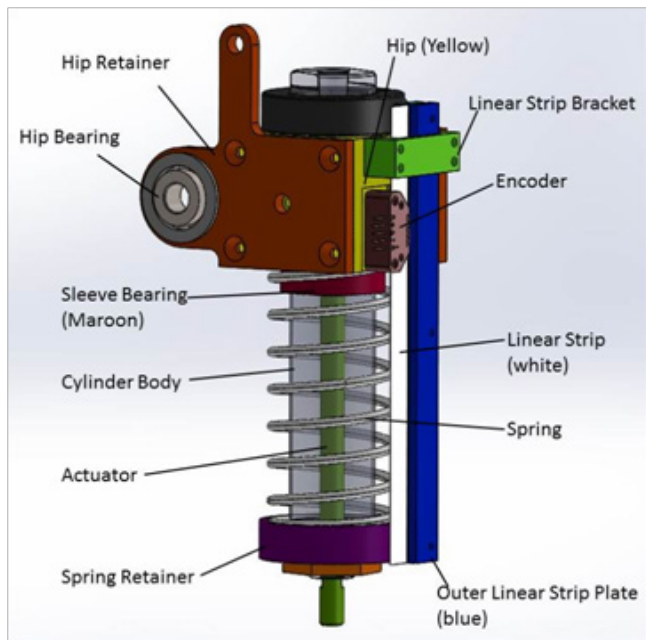

Figure 17 CAD drawing of one leg of the 4 DOF hopping robot.

\section{Simulation \& experiments}

A variety of 2 DOF planer models were created in Working Model 2D. The models consisted of two spring actuator legs connected by a link of half the estimated weight of the robot and the length equivalent to the longest length of the robot. The legs were fixed in a perpendicular position relative to the body for all of the models. In simulations where no disturbances were introduced, the model was stable and quickly converged on a steady state (Figure 18). In this model an unintended consequence of the new leg design was realized. The model showed that as the actuators fired and the mass would begin to move upward vertically, the springs were compressing as well, absorbing some of the energy that was being added to the system. It appeared that this affected the efficiency of the robot but did not detract from its' stability characteristics.

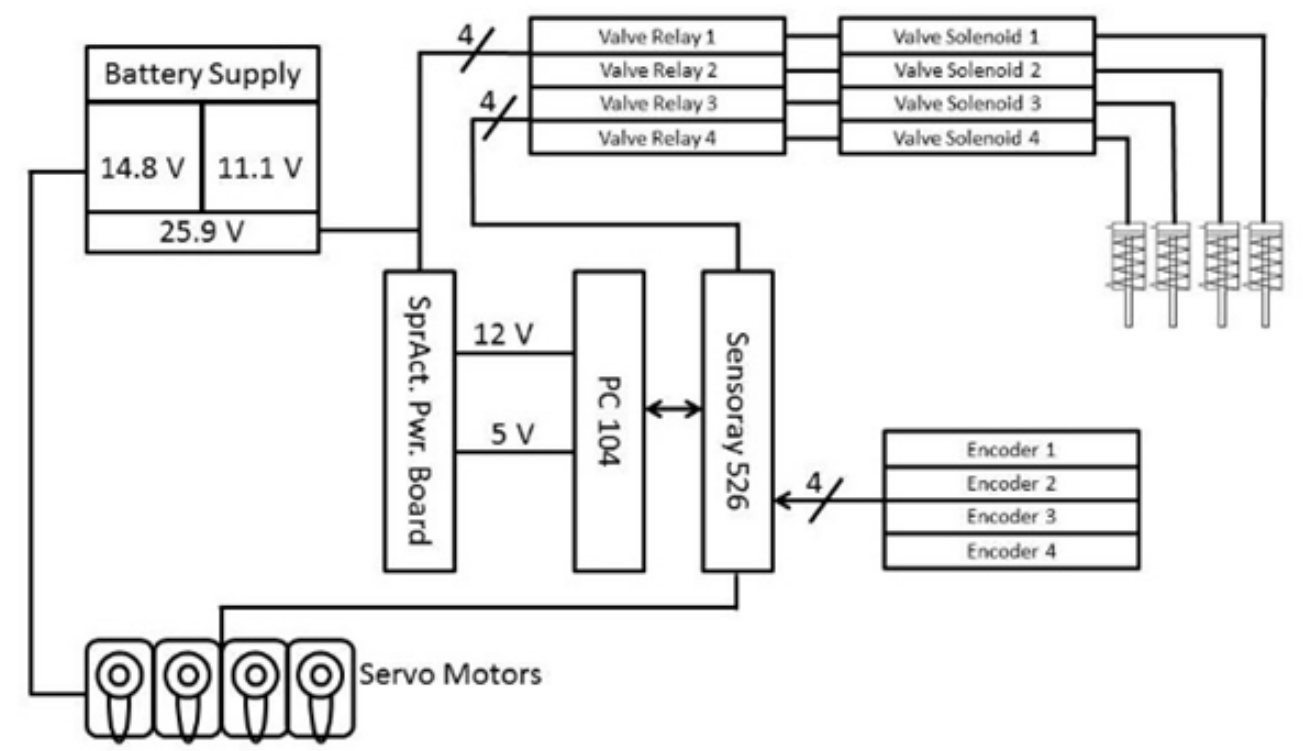

Figure 18 Block diagram showing component configuration of the quadruped system.

In another simulation a disturbance was presented by adding a block to the otherwise flat ground. The block can be seen in Figure 19 on the left hand side of the figure. To start the model, one leg of the robot was placed on top of the block. This made the robot hop to the left and alternatively back to the right again. After running five seconds, the robot encountered the disturbance again; landing on the block for two consecutive hops. This forced the model to go unstable toppling over to an inverted position. This result was not necessarily unexpected as it was assumed that a disturbance of large enough size and duration could produce an instability. It is important to note that there was no trajectory objective programmed into the control software. The model was programmed with the Phase Oscillator Controller with the objective of hopping. 


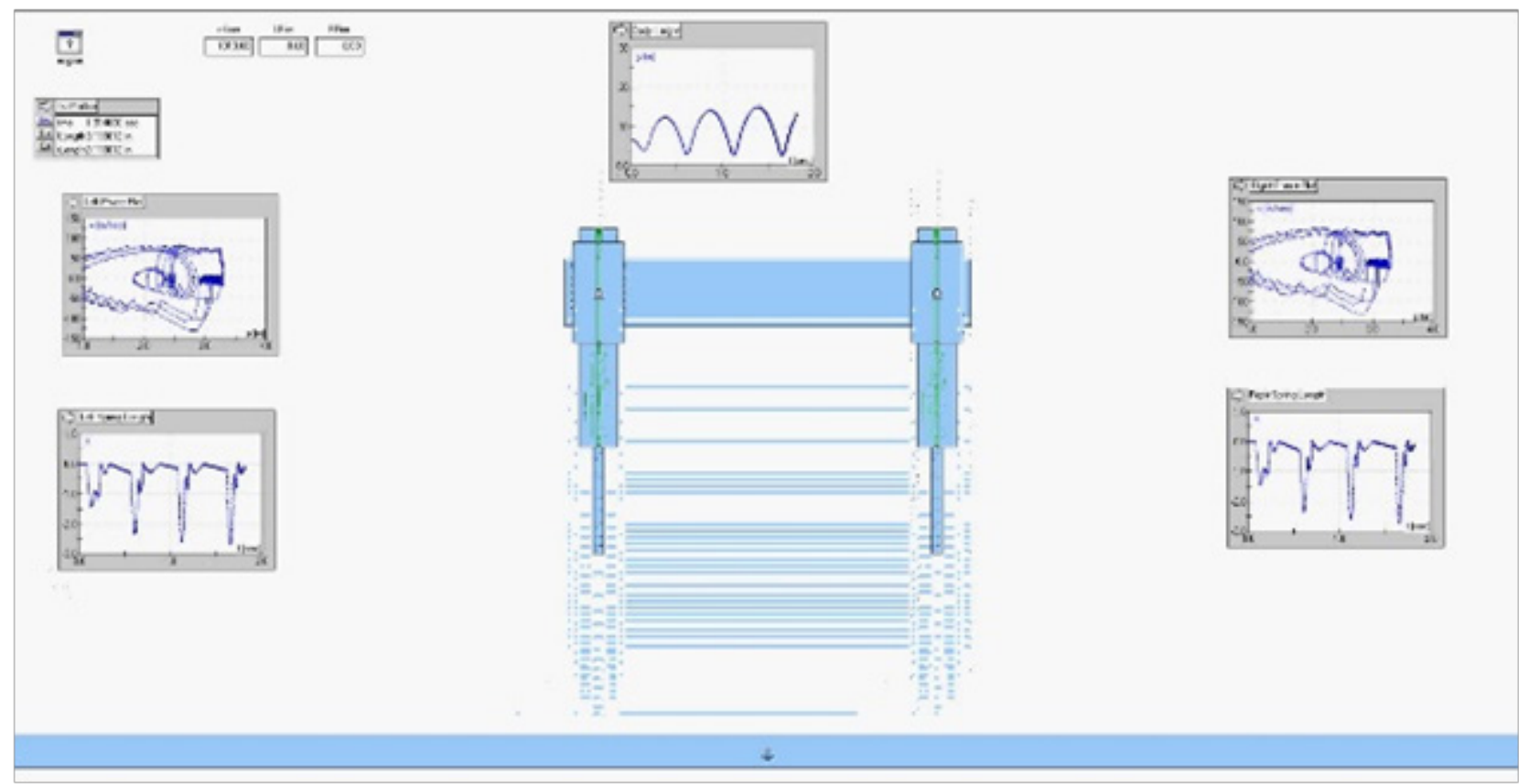

Figure 19 Working model simulation with no disturbance and theta equals zero.

In actual experiments, encoders on robot were used to collect four sets of data, see Table 1. Three sets were collected using one encoder to determine when to activate all the actuators. All four encoders collected position data. The fourth trial used all four encoders to determine the actuation timing of each individual, respective leg. Again, all four encoders collected position data for each leg. The phase portraits for the spring show that while the robot moved toward a steady state oscillation there were some full compressions of the springs. Approximately $0.15 \mathrm{~m}$ represented a full compression of the spring. These events were the extreme case and were generally dampened on the subsequent hops. In Figure 20, a clear bounded 'limit' cycle seems to emerge from the graph. It should also be noted that the spring compression did not follow a regular pattern. The compressions increased or decreased due to the attitude of the robot. When a leg was the first to return to the ground, that particular leg would tend to compress more than the others. The last leg to hit was conversely the leg that experienced the least compression in its spring. Figure 21 displays the test results from a trial that introduced a disturbance. The disturbance was created by manually nudging or hitting robot while hopping. It can be observed that despite of disturbance the robot retuned to steady hopping (Figure 22).

Table I Completed tests of the quadruped hopper.

\begin{tabular}{llll}
\hline $\begin{array}{l}\text { Quadruped } \\
\text { Hopper }\end{array}$ & $\begin{array}{l}\text { Transient } \\
\text { Response }\end{array}$ & $\begin{array}{l}\text { Steady } \\
\text { State }\end{array}$ & Disturbance \\
\hline One Encoder & $\mathrm{X}$ & $\mathrm{X}$ & $\mathrm{X}$ \\
All Encoders & $\mathrm{X}$ & $\mathrm{X}$ & $\mathrm{X}$ \\
\hline
\end{tabular}

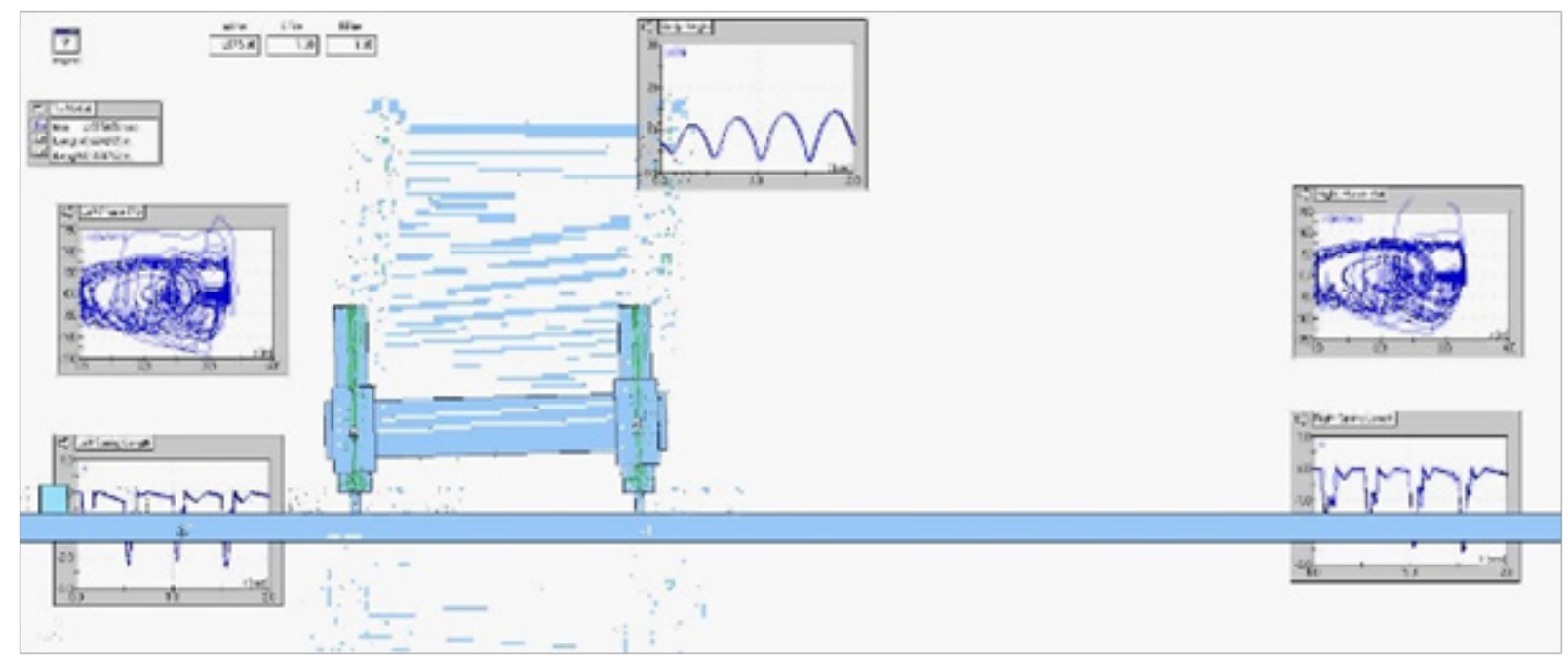

Figure 20 Working Model simulation with a disturbance, theta was non-zero. 


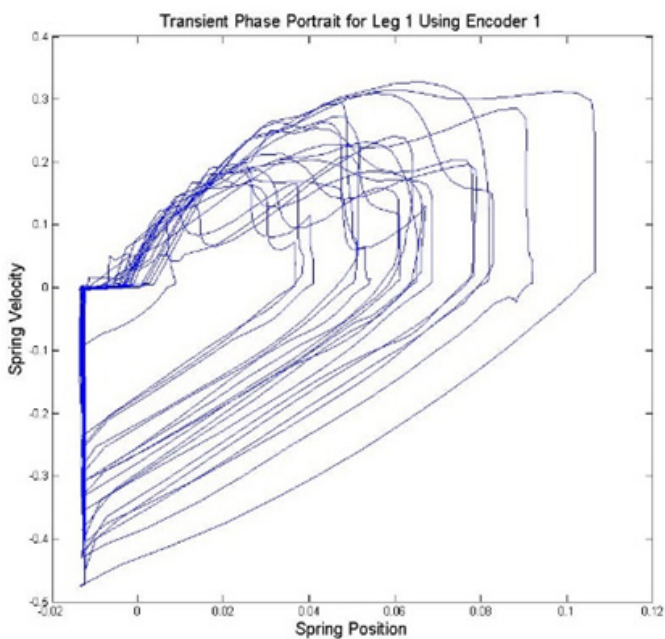

Figure 2 I Transient phase portrait of leg I while actuation was controlled from this phase plot.

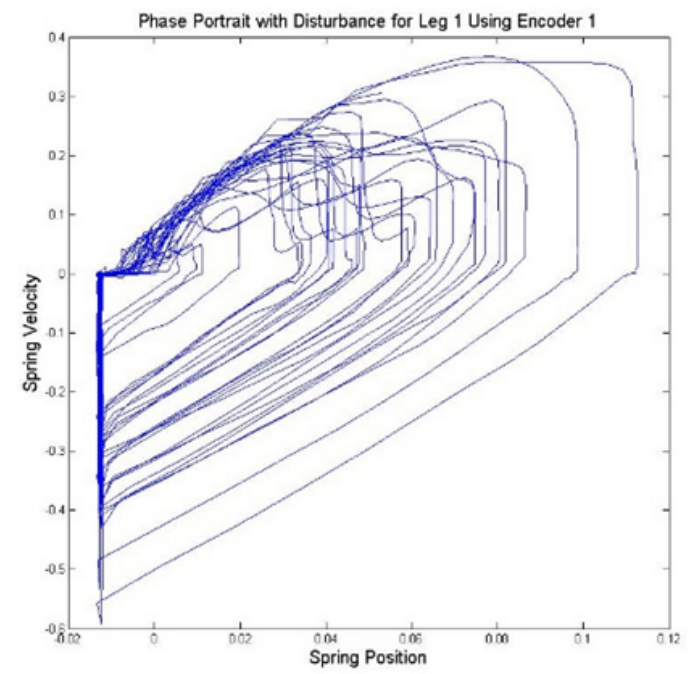

Figure 22 The phase portrait of leg I with an added disturbance while actuation was controlled from the phase plot of leg $\mathrm{I}$.

\section{Conclusion}

In this work, an application of phase oscillator for quadruped hopping robot was investigated. The controller was adaptive and was able to drive the system to a controlled limit cycle despite of perturbation. This control strategy is easier to implement in a real time. This control loop can execute at $100 \mathrm{~Hz}$ on a low cost microcontroller. Another advantage of this oscillator was that it learned the amplitude and frequency of the external periodic signal quickly. The system was based in the entrainment or phase lock of the external signal and the oscillator. In future, we are planning to implement adaptive hopf oscillator in hopping robot. This oscillator aims at providing assistance to periodic motions. It can be used to synchronize the oscillator with external periodic signal and then to estimate the states of the system. The estimated states will used to compute the control signal and then force the system to follow the behavior.

\section{Acknowledgments}

None.

\section{Conflict of interest}

The authors declare that there are no conflicts of interest.

\section{Funding}

None.

\section{References}

1. Naidoo Y, Stopforth R., Bright G. Development of an UAV for Search \& Rescue Applications. IEEE. 2011. p. 1-6.

2. Defense Advanced Research Project Agency (DARPA). "Digitizing SQUAD X: Sensing, Communications, Mission Command, and Soldierworn Backbone, 2014.

3. Nelson G. Where is Google's Car Going? Automotive News, 2015;89(6654):1.

4. Hong DW. Analysis and Visualization of the Contact Force Solution Space for Multilimbed Mobile Robots," Ph.D. thesis (Order No. 3099796). Lafayette: Purdue University; 2015.

5. Hong DW, Ingram M, Lahr D. Whole Skin Locomotion Inspired by Amoeboid Motility Mechanisms. ASME Journal of Mechanisms and Robotics. 2009;1(1):011015.

6. Hong DW, Cipra RJ. Optimal Contact Force Distribution for MultiLimbed Robots. ASME Journal of Mechanical Design. 2006;128(3):566573

7. Sayyad A, Seth B, Seshu P. Single-Legged Hopping Robotics Research A Review. Robotica. 2007;25(5):587-613.

8. Tokhi MO, Virk GS, Hossain MA. Climbing and Walking Robots. Proceedings of the 8th International Conference on Climbing and Walking Robots and the Support Technologies for Mobile Machines. 2006.

9. Wetter green D, Thorpe C, Whittaker WL. Exploring Mount Erebus by Walking Robot. Robotics and Autonomous Systems. 1993;11:171-185.

10. Murphy RR. Trial by Fire [rescue Robots]. IEEE Robotics Automation Magazine. 2004;11(3):50-61.

11. Aguirre Ollinger G, Colgate JE, Peshkin MA. A 1-DOF Assistive Exoskeleton with Virtual Negative Damping: Effects on the Kinematic Response of the Lower Limbs. IROS 2007, IEEE/RSJ International Conference on Intelligent Robots and Systems, 2007.

12. Holgate MA, Bohler AW, Sugar TG. Control Algorithms for Ankle Robots: A Reflection on the State-of-the-Art and Presentation of Two Novel Algorithms. IEEE. 2008. p. 97-102.

13. Holgate MA, Sugar TG, Bohler AW. A novel control algorithm for wearable robotics using phase plane invariants.. IEEE International Conference on. Japan: Kobe; 2009. p. 3845-3850.

14. Hogan N. Impedance Control: An Approach to Manipulation. American Controls Conference. 1984. p. 304-313.

15. New P, Wheeler C, Sugar TG. Robotic Hopper Using Phase Oscillator Controller. ASME 2014 International Design Engineering Technical Conferences and Computers and Information in Engineering Conference, ASME, United States: Buffalo; 2014.

16. Sugar TG, Bates A, New P, et al. Limit Cycles to Enhance Human Performance based on Phase Oscillators. Journal of Mechanisms and Robotics. 2015;1(0110011):1-8.

17. Raibert Marc. Legged Robots, Communication of the ACM. 1986;29(6):499-514.

18. Nof SY. Handbook of Industrial Robotics. New York: John Wiley; 1999. p. 148 . 
19. Zhuang HC, Gao HB, Deng ZQ, et al. A Review of Heavy-Duty Legged Robots. Sci China Tech Sci. 2014;(57):298-314.

20. Carbone G, Ceccarelli M. Legged Robotic Systems. INTECH Open Access Publisher; 2005. pp. 557-561.

21. Nelson G, Blankespoor K, Raibert M. Walking BigDog: Insights and Challenges from Legged Robotics. Journal of Biomechanics. 2006;39(S 1):S360.

22. Ma J, Susca S, Bajracharya M, et al. Robust Multi-Sensor, Day/ Night 6-DOF Pose Estimation for a Dynamic Legged Vehicle in GPSDenied Environments. Robotics and Automation (ICRA), 2012 IEEE International Conference on. IEEE. 295 p.

23. Wilcox BH. ATHELETE: A Cargo and Habitat Transporter for the Moon Aerospace Conference, 2009 IEEE. IEEE; 2009. 385 p.

24. Ishi H, Koga H, Obokawa Y, et al. Path Generator Control System and Virtual Compliance Calculator for Maxillofacial Massage Robots. International Journal of Computer Assisted Radiology and Surgery. 2010;5:77-84.

25. Haeufle DBF, Grimmer S, Seyfarth A. The Role of Intrinsic Muscle Properties for Stable Hopping-Stability is Achieved by the ForceVelocity Relation. Bioinspiration \& Bimimetics. 2010;5(2):1-11.

26. Zhou X, Bi S. A Survey of Bio-Inspired Compliant Legged Robot Designs. Bioinspiration \& Bimimetics. 2012;7(3):1-20.
27. Mochon S, McMahon TA. Ballistic Walking: An Improved Model. Mathematical Biosciences. 1980;52(3-4):241-260.

28. Formal'sky A, Chevallereau C, Perrin B. On Ballistic Walking Locomotion of a Quadruped. The International Journal of Robotics Research. 2000;19(8):743-761.

29. Kalveram KT, Haufle D, Grimmer S, et al. Energy management that generates hopping. Comparison of virtual robotic and human bouncing. Proceedings of SIMPAR 2010 Workshops. International Conference on Simulation, Modeling and Programming for Autonomous Robots. 2010. pp 147-156.

30. Haeufle DFB, Gunther M, Bayer A, et al. Hill-Type Muscle Model with Serial Dampening and Eccentric Force-Velocity Relation. Journal of Biomechanics. 2014;47(6):1531-1536.

31. Winters JM, Woo SLY. Multiple Muscle Systems: Biomechanics and Movement Organization. New York: Springer-Verlag; 1990. pp. 69-93.

32. Graham D, McRuer DT. Phase Plane Analysis. In: Analysis of Nonlinear Control Systems, Dover Press; 1971. 48 p.

33. Redkar S, Liu J, Sinha SC. Stability and robustness analysis of linear timeperiodic system subjected to random perturbations. Communications in Nonlinear Science and Numerical Simulations. 2012;17(3):1430-1437. 